\title{
Thrombophilia in hyperhomocysteinemia
}

\author{
Vishal Sharma • Mukul P. Agarwal • Subhash Giri • \\ Srimanta K. Sahu $\cdot$ Ujjawal Roy
}

Received: 10 December 2009 / Accepted: 31 May 2010/Published online: 20 August 2010

(C) Springer-Verlag London Ltd 2010

A 23-year-old otherwise healthy male presented with development of painful blackish discoloration of the right thumb and index finger and of the right big toe and area over the ankle (Figs. 1 and 2), as well as painful swelling of both lower limbs, especially on the right side (Fig. 3). Homans'sign was present bilaterally. His past history was not significant. Routine investigations, including complete blood count, coagulation profile, chest roentgenogram and ultrasound of the abdomen, were normal.

The patient presented with evidence of an increased tendency to thrombosis, both venous and arterial. Doppler ultrasonography confirmed bilateral lower limb deep venous thrombosis with complete occlusion of the right radial artery. His echocardiography and HIV serology were

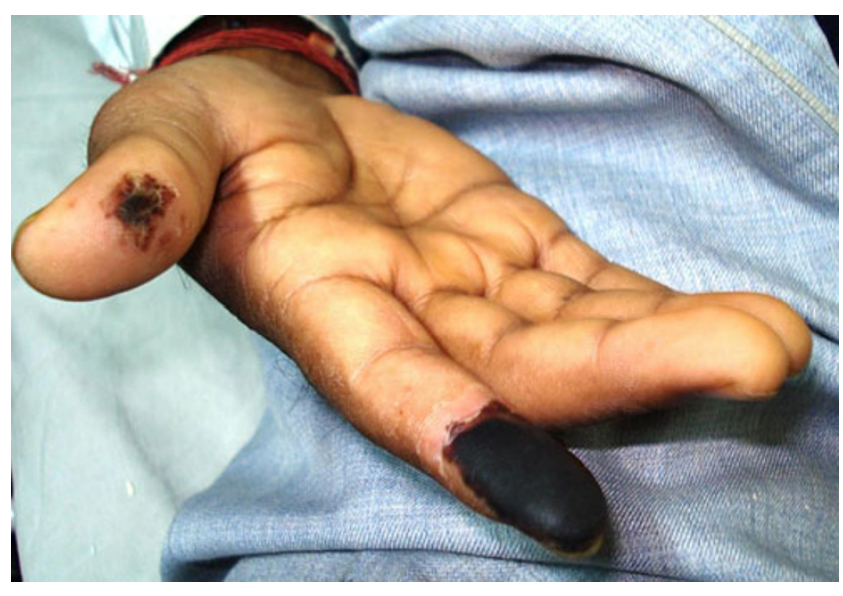

Fig. 1 The patient demonstrated a blackish discoloration of the right thumb and index finger

V. Sharma $(\bowtie) \cdot$ M. P. Agarwal $\cdot$ S. Giri $\cdot$ S. K. Sahu $\cdot$ U. Roy

Department of Medicine, University College of Medical Sciences, Delhi, India

e-mail: docvishalsharma@gmail.com

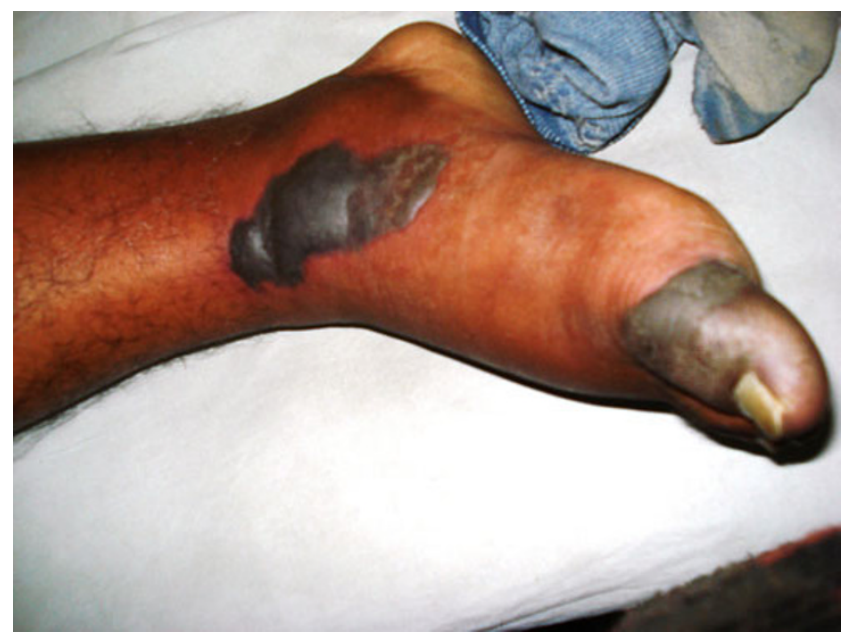

Fig. 2 The patient demonstrated blackish discoloration of the right big toe and the area over the ankle

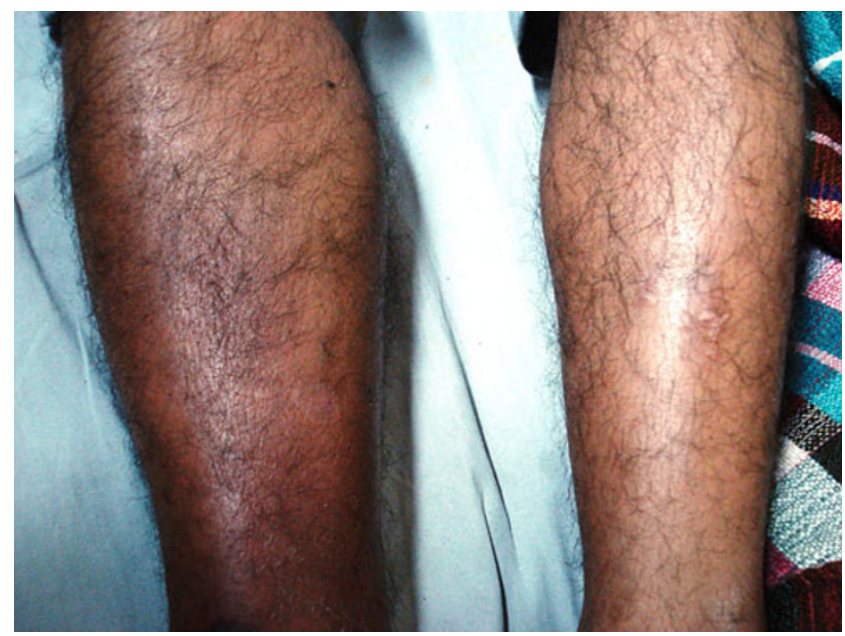

Fig. 3 The patient demonstrated swollen lower limbs, especially on the right side 
normal. His serum homocysteine levels were elevated at $52 \mu \mathrm{mol} / 1$ (normal, $4.4-10.8 \mu \mathrm{mol} / \mathrm{l}$ ). The patient was managed with enoxaparin, warfarin and folic acid. Within a week, the swelling of the lower limbs improved, and the patient was discharged on warfarin.
Only a few causes are known to cause both arterial and venous thrombosis, including elevated homocysteine levels, antiphospholipid syndrome, polycythemia vera, paroxysmal nocturnal hemoglobinuria, underlying malignancy and hormonal therapy. 